\title{
¿CÓMO LAS COMPETENCIAS ACTITUDINALES AYUDAN A CONSEGUIR UN ADECUADO APRENDIZAJE EN DISCENTES?
}

\author{
Elmina Matilde Rivadeneira Rodríguez \\ Máster en Derechos de la Infancia y la Adolescencia en España \\ Doctora en Orientación y Formación de Competencias en España \\ Postdoctorado en Metodología de Investigación en Estados Unidos \\ elminar@hotmail.com
}

\section{Cómo citar este artículo}

Rivadeneira, E. (2013) ¿Cómo las competencias actitudinales ayudan a conseguir un adecuado aprendizaje en discentes? Espiral, Revista de Docencia e Investigación. 3 (1) 57 - 64

\section{Resumen}

Al referirnos a las competencias actitudinales, estamos refiriéndonos al "SABER SER/SABER ACTUAR". En primer momento, se realiza una revisión de las aportaciones con relación a habilidades actitudinales del docente - discente, destacando la importancia de las relaciones ínter e intrapersonales para evitar la desmotivación, la falta de implicación, de confianza en sí mismo y en los adultos, la escasa valoración de sí mismo, y fomentar la comprensión de los docentes frente a los conflictos que se presentan en el aula. A continuación, se presentan los siguientes valores actitudinales positivos: proactividad, creatividad, confiabilidad, flexibilidad, resiliencia, empatía, confianza en sí mismo, solidaridad, comprensión, que permitan mejorar las relaciones ínter e intrapersonales para mejora los desempeños de los discentes, y los valores actitudinales negativos (candados mentales). Finalmente, se hace mención sobre la importancia de la creación de un clima favorable para conseguir el desarrollo y dominio del aprendizaje de los discentes haciendo uso del trabajo en equipo.

Palabras clave: competencias actitudinales, desarroIlo, dominio, valores, interpersonales, intrapersonales, desmotivación, integración, inteligencia emocional, trabajo en equipo.

\footnotetext{
Abstract

When referring to the attitudinal skills, we are referring to "KNOW BE / KNOW ACT". . At first, we review the relative contributions of the attitudinal skills teacher - learner, emphasizing the importance of inter - intra to avoid discouragement, lack of involvement, trust in you and in adults, the low valuation of himself, and to promote understanding of teachers face the conflicts that arise in the classroom. Then the following values are positive attitudinal: proactivity, creativity, reliability, flexibility, resilience, empathy, confidence, solidarity, understan-
}

ding, to improve international relations - intrapersonal improves performance of learners, and negative attitudinal values (mental locks). Finally, mention is made of the importance of creating a favorable climate for the development and mastery learning of learners making use of teamwork.

Keywords: attitudinal skills, development, domain, values, interpersonal, intrapersonal, motivation, integration, emotional intelligence, teamwork.

\section{INTRODUCCIÓN}

¿Puede el cambio de actitud mejorar las relaciones interpersonales entre docentes y discentes?

Las competencias actitudinales (saber ser / saber actuar) son características que poseen determinadas personas que hacen que su comportamiento y desempeño sea especialmente satisfactorio en el entorno familiar, social, laboral, educativo, profesional y demás. Según Le Boterf (2001) el ser profesional conlleva saber actuar y reaccionar con pertinencia, saber combinar los recursos y movilizarlos en un contexto, saber transferir, saber aprender, así como saber comprometerse.

\section{- El docente}

El nuevo perfil del siglo XXI, asume la necesidad de plantear nuevas características, es decir, otras bases epistemológicas para el desempeño del docente: proactivo, creativo, confiable, flexible, optimista, solidario, comprensivo, ca- 
paz de ejercer su acción pedagógica en los diferentes niveles y modalidades de aprendizaje. La sociedad del futuro exigirá al docente enfrentarse con situaciones difíciles y complejas: grupos extremadamente heterogéneos, acceso a puestos en forma provisoria, rápida y permanente; evolución cultural y social, especialmente en los jóvenes en quienes existe la sensación que no hay futuro y la pérdida de interés al sentido del saber o el aprender (Bar, 1999).

Miras (2001) sostiene que los aspectos relacionados con el clima social y emocional han sido tratados como si fueran emocionalmente neutros, es decir como que no tuvieran importancia. El desarrollo intrapersonal e interpersonal, como parte motivacional del aprendizaje, conlleva a mirar en su contexto interno externo a docentes y discentes para interactuar en el mundo real (Gardner, 2003). Fierro (2003) reafirma lo expresado por Goleman al exponer que el docente es un ser humano con un mundo interior y exterior, por lo tanto, la práctica docente es una práctica humana, donde debe ser entendido como individuo con cualidades, características, defectos, potencialidades y dificultades; con ideales proyectos, motivaciones, imperfecciones. Dada su individualidad, las decisiones adquieren un carácter particular. De hecho el postmodernismo invita a los profesionales a desarrollar y dominar capacidades conceptuales, procedimentales y actitudinales que le permitan interactuar con afecto en el momento de la práctica pedagógica.

FIGURA 1. Perfil del docente basado en las competencias

\begin{tabular}{|c|c|}
\hline Competencias ínter e intrapersonales (saber ser) & $\begin{array}{l}\text { Competencias sociales } \\
\text { (Saber actuar en convivencia) }\end{array}$ \\
\hline $\begin{array}{l}\text { - } \quad \text { Asume responsablemente el riesgo de sus opciones } \\
\text { - } \quad \text { Asume los cambios crítica y creativamente } \\
\text { Desarrolla interés por comprender y profundizar } \\
\text { diferentes aspectos de la realidad } \\
\text { - } \quad \text { Vive en coherencia con los valores que propone } \\
\text { - } \quad \text { Mantiene independencia sin perder apertura }\end{array}$ & $\begin{array}{l}\text { - } \quad \text { Genera respuestas adecuadas para el bienestar colectivo } \\
\text { - } \quad \text { Desarrolla las capacidades lúdicas de los estudiantes } \\
\text { - } \quad \text { Analiza e interpreta en equipos interdisciplinarios, la } \\
\text { - } \quad \text { realidad compleja, para plantear soluciones } \\
\text { deconoce, práctica y divulga la defensa de la salud, los } \\
\text { - } \quad \text { Practica y fomenta la responsabilidad solidaria, la } \\
\text { participación y la equidad } \\
\text { Se compromete con los problemas y aspiraciones de la } \\
\text { comunidad }\end{array}$ \\
\hline
\end{tabular}

Fuente: Elaboración propia: Rivadeneira (2013). Adaptada de Calvis (2007)

Irigoin y Vargas (2002:47) definen la competencia como "combinación integrada de conocimientos, habilidades y actitudes que se ponen en acción para un desempeño adecuado en un contexto dado. Más aún, se habla de un saber actuar movilizando todos los recursos". Las competencias integran diferentes capacidades (cognoscitivas, psicomotoras y afectivas) que, combinadas entre sí y movilizadas adecuadamente, per- miten la realización de un desempeño profesional efectivo (Barreira, 2003).

El docente debe estar abierto e inmerso en los cambios para orientar y estimular aprendizaje, desarrollar el liderazgo, la capacidad de interactuar armónicamente con las personas y resolver conflictos, así mismo desarrollar la capacidad de aprender a aprender, la capacidad de innovar, de automotivarse, ser capaz de ponerse en el lugar de los demás (empatía). 
FIGURA 2. Saberes

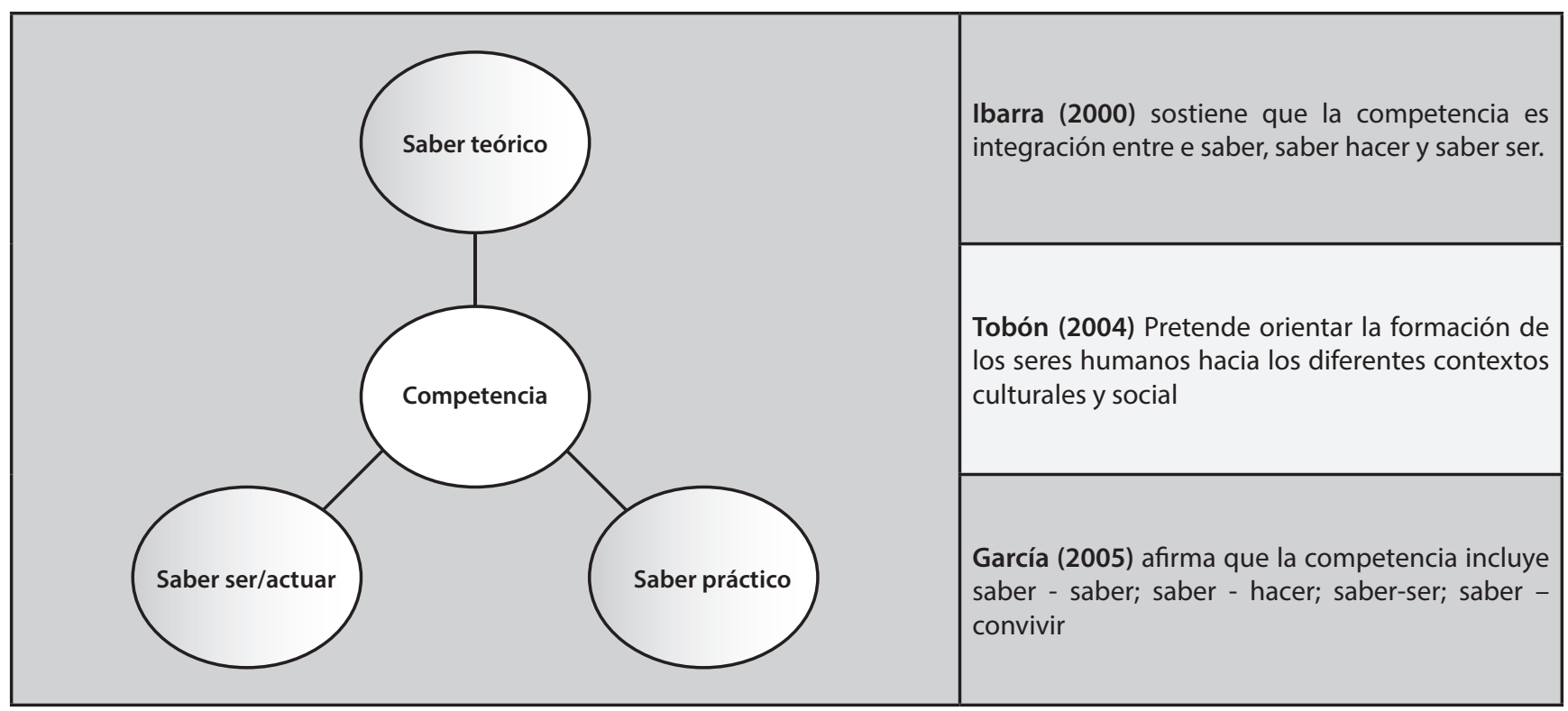

Fuente: Elaboración propia: Rivadeneira (2013). Adaptado Ibarra (2000), Tobón (2004) y García (2005)

\section{- El discente}

La transformación en la formación integral puede alcanzarse al desarrollar las competencias sociales, ínter e intrapersonales del estudiante, en un componente general donde se estimule la comprensión del otro y la percepción de las formas de interdependencia y prepararse para resolver conflictos respetando los valores de cada uno (Calvis, 2007).

Braslaysky (1998) afirma que no hay sujeto sin competencia, ni humanos sin personalidad. Simplemente, hay seres humanos que no encontraron en la escuela y posiblemente desde la familia y la sociedad, los espacios culturales y formativos que le permitieran el despliegue de sus capacidades intelectuales, sus gustos y afectos, sentir la emoción del descubrimiento.

En otras palabras el ambiente positivo condiciona las relaciones entre docente - discente $y$, a su vez, mejora el proceso de motivacion, autodominio, creatividad, autonomía y aprendizaje de los discentes.
- El ser humano desde una perspectiva integradora: emociones positivas y negativas

Mediante esta perspectiva se considera al ser humano como un agente activo capaz de construir sus propia realidad. McLeod, (1989) considera que el dominio efectivo de la educación no es solamente cognición, sino que se debe tomar en consideración los sentimientos, actitudes, creencias y emociones. Esta aportación se sustenta en estudios que sostienen que experimentar emociones positivas favorece el pensamiento creativo para la solución de problemas interpersonales. Fredrickson (1998) destaca cuatro tipos de emociones positivas: alegría, interés, amor y satisfacción. Además el autor sostiene que las emociones positivas provocan cambios en las esferas conductuales (Fredrickson, 2001). En la actualidad se ha dado énfasis al estudiar y de incluir la inteligencia emocional en el ámbito escolar.

Las emociones, permiten al discente ser más eficaz cuando puede escuchar con tolerancia, y, a su vez, verse como un ser humano imperfec- 
to que no siempre su actuación es con conocimiento y puede reconocer sus emociones negativas como: enojo, desinterés, aburrimiento, ansiedad, y miedo entre otras. De hecho este es el momento en que el docente se acepte a sí mismo, de ello depende que decida cambiar de actitud el discente. Deacuerdo con (Ekman, 1999) las emociones positivas y negativas se diferencian entre ellas por su expresión fisiológica. Las emociones básicas negativas (temor, ira, aversión o hastío, tristeza y desprecio); mientras que las emociones básicas positivas (diversión, orgullo en proeza, satisfacción, alivio y contexto.

\section{DESARROLLO}

¿De qué manera la aplicación de los valores actitudinales ayudan a erradicar sentimientos de incompetencia en los discentes?

En este apartado se propone analizar los valores actitudinales positivos y negativos que ayudan a mejorar las relaciones interpersonales entre docente - discente.

En sentido Delors (1996) menciona los pilares fundamentales de la educación: aprender a conocer, aprender hacer, aprender a convivir, aprender a ser, con esta aportación coincide (Tedesco, 1995).

FIGURA 3. Pilares fundamentales

\begin{tabular}{|c|c|c|c|}
\hline $\begin{array}{l}\text { Aprender } \\
\text { a conocer }\end{array}$ & $\begin{array}{c}\text { Aprender } \\
\text { a hacer }\end{array}$ & $\begin{array}{l}\text { Aprender } \\
\text { a convivir }\end{array}$ & $\begin{array}{l}\text { Aprender } \\
\text { a ser }\end{array}$ \\
\hline $\begin{array}{l}\text { Desarrollo de operaciones } \\
\text { analíticas, relacionales } \\
\text { e integradoras, de } \\
\text { acuerdo a los niveles de } \\
\text { desarrollo y para construir } \\
\text { los correspondientes } \\
\text { instrumentos del } \\
\text { conocimiento (de nociones } \\
\text { a categorías, teorías, } \\
\text { concepciones) }\end{array}$ & $\begin{array}{l}\text { Implica operaciones } \\
\text { efectivas de actuación, } \\
\text { ejecución y de } \\
\text { transformación, para poder } \\
\text { influir sobre el propio } \\
\text { entorno (relación entre la } \\
\text { teoría y la práctica) }\end{array}$ & $\begin{array}{l}\text { Capacidad de expresión, afecto, } \\
\text { comunicación, valoración, } \\
\text { participación, concertación y } \\
\text { afectividad, para participar y } \\
\text { cooperar con los demás en todas } \\
\text { las actividades humanas.(relación } \\
\text { entre saber comunicar, demostrar, } \\
\text { expresar nuestros afectos, respeto, } \\
\text { consideración a los demás) }\end{array}$ & $\begin{array}{l}\text { Recoge elementos de los } \\
\text { tres anteriores, para que } \\
\text { aflore la personalidad y se } \\
\text { esté en capacidad de obrar } \\
\text { con autonomía, juicio y } \\
\text { responsabilidad, personal } \\
\text { (saber ser / saber actuar) }\end{array}$ \\
\hline
\end{tabular}

Fuente: Elaboración propia: Rivadeneira (2013). Adaptada Delors (1996)

\section{- Relación entre actitudes - valores}

La educación es un factor importante para desarrollar las actitudes permanentes y fortalecer los valores. El empoderamiento de los valores y las actitudes se refleja en los desempeños, mediante el proceso de sociabilización y la aplicación de reglas en la convivencia de los individuos para saber ser/actuar. La educación pretende potenciar los valores en las personas y facilitar su adaptación cultural, social, personal. Los valores constituyen el polo objetivo que califica y da sentido a las actitudes (Marín, 1976)

Además de atender en primer lugar las actitudes (internas - autoconcepto), en muchos casos la resistencia a la frustración, tranquilidad permanente, y demás, y por otra parte, promover las actitudes personales (externas) en contexto natural o social (Coopersmith y Feldman, 1974). Algunas investigaciones corroboran estas relaciones autoconcepto y relaciones interpersonales (Fitts, 1970).

\section{- Actitudes positivas y negativas}

Pocas veces ha sido tratado las actitudes en una forma sistemática. Por ello, la preocupación aumenta a medida que comprobamos de la falta de planteamientos tradicionales para alcanzar los objetivos educativos que una sociedad actual exige con relación a los nuevos retos y transformaciones. 
FIGURA 4. Saber ser/saber actuar

\begin{tabular}{|l|l|}
\hline \multicolumn{1}{c|}{\begin{tabular}{c}
\multicolumn{1}{c|}{$\begin{array}{c}\text { Actitud positiva } \\
\text { (Mejora el autoconcepto) }\end{array}$} \\
Proactividad
\end{tabular}} & \multicolumn{1}{c|}{$\begin{array}{c}\text { Actitud negativa } \\
\text { (Candados mentales) }\end{array}$} \\
Creatividad & $\begin{array}{l}\text { Reactiva } \\
\text { Ira } \\
\text { Flexibilidad }\end{array}$ \\
Confianza en sí mismo & Miedo \\
Resilencia & Disgusto \\
Optimismo & Envidia \\
Empatía & Resentimiento \\
Solidaridad & Egoísmo \\
Comprensión & Sentimiento de culpa \\
\hline
\end{tabular}

Fuente: Elaboración propia: Rivadeneira (2013). Adaptado de Goleman (1995), Calderón, Hess, Pérez (1998); Steven (2003)

Goleman (1995) afirma que las virtudes como (esperanza, fe, coraje, certeza, indulgencia, ecuanimidad) y los vicios clásicos (sentimiento de duda, complacencia, indolencia, apatía).

\section{Clima favorable para desarrollar el aprendizaje en los discentes}

Un buen clima de aprendizaje favorece en el afianzamiento del autoconcepto, autoestima y la seguridad de sí mismo, a medida que se trasmite aliento frente a las tareas complejas, se valora los logros, se les ayuda a entender sus fracasos como nuevas oportunidades. Coll y Miras (1990) sostienen que el "profesor ideal" otorga una importancia primordial a los aspectos afectivos y de relación interpersonal (disponibilidad, respeto, simpatía, nivel de atención personal y demás). Cada vez se ha defendido el aprendizaje de los discentes se debe mayoritariamente en los elementos externos (familia, cultura, incidencia del docente y contenido entre otros) y pocas veces se ha destacado la importancia de los valores oncogénicos: motivación, actitudes, intereses, y otros. Oñativia (1973) analiza los intereses y las actitudes y destaca que se ha hablado mucho de los intereses de los escolares, pero se ha hecho poco por fomentar el desarrollo de las actitudes.

En los momentos actuales se reclama la necesidad de crear un clima emocional positivo en el aula entre las condiciones para desarrollar un buen aprendizaje (Glasser, 1984) o centrado en el aprendiz, como mencionan McCombs y Whisler (1997), además señalan que el aprendizaje del estudiante se produce mejor en un entorno positivo; en el que se puedan establecer relaciones interpersonales positivas y se dan unas condiciones de comodidad y orden; un contexto en el que el aprendiz se sienta apreciado, reconocido, respetado y validado.

La creación de un clima relajado y con buen humor en sus aulas consigue que el alumnado también se relaje, demuestre lo mejor de sí mismo y observe al profesor o la profesora "como un aliado y amigo, en vez de entenderlo como miembro de una especie enemiga" (Fontana, 1994,146). En este contexto, el alumnado está también mucho más dispuesto a cooperar y es improbable que se produzcan incidentes que den lugar a problemas de control en el aula.

El docente, guía, formador, orientador debe crear un ambiente de seguridad psicológica y emocional para facilitar la expresión de sus emociones, incertidumbres, dudas, temores, miedos propia de su formación; son los docentes que acompañan a los discentes con una actitud respetuosa, responsable y comprometida en la aventura del aprendizaje. La aplicación de las habilidades sociales es la base para las relaciones interpersonales. En el momento que entendemos y comprendemos al otro su manera de pensar de actuar, sus sentimientos, se utiliza la comunicación (Aldaz, 2007). 
FIGURA 5. Habilidades sociales

\begin{tabular}{|l|l|}
\hline Influencia & Proponer tácticas efectiva de persuasión \\
\hline Comunicación & Saber escuchar atentamente \\
\hline Manejo de conflictos & Saber negociar resolver los desacuerdos \\
\hline Liderazgo & Capacidad de inspirar y guiar los individuos y al grupo \\
\hline Catalizador del cambio & Iniciador o administrador de las situaciones nuevas \\
\hline Constructor de lazos & Alimentar y reforzar las relaciones interpersonales \\
\hline Colaboración y cooperación & Trabajos con otros \\
\hline Capacidad de equipo & \\
\hline
\end{tabular}

Elaboración propia: Rivadeneira (2013). Adaptado Aldaz (2007)

2. Valores actitudinales positivos/negativos $y$ trabajo en equipo en el aula para conseguir el aprendizaje de los discentes

Tradicionalmente la educación hacia énfasis en la instrucción y en la concepción que el estu- diante era una ente pasivo frente a la recepción de información que transmitía en docente. En los actuales momentos se pone énfasis en el aprender por lo tanto convierte al discente en sujeto activo de su propio desarrollo conceptual, procedimental y actitudinal (ser/actuar).

FIGURA 6.

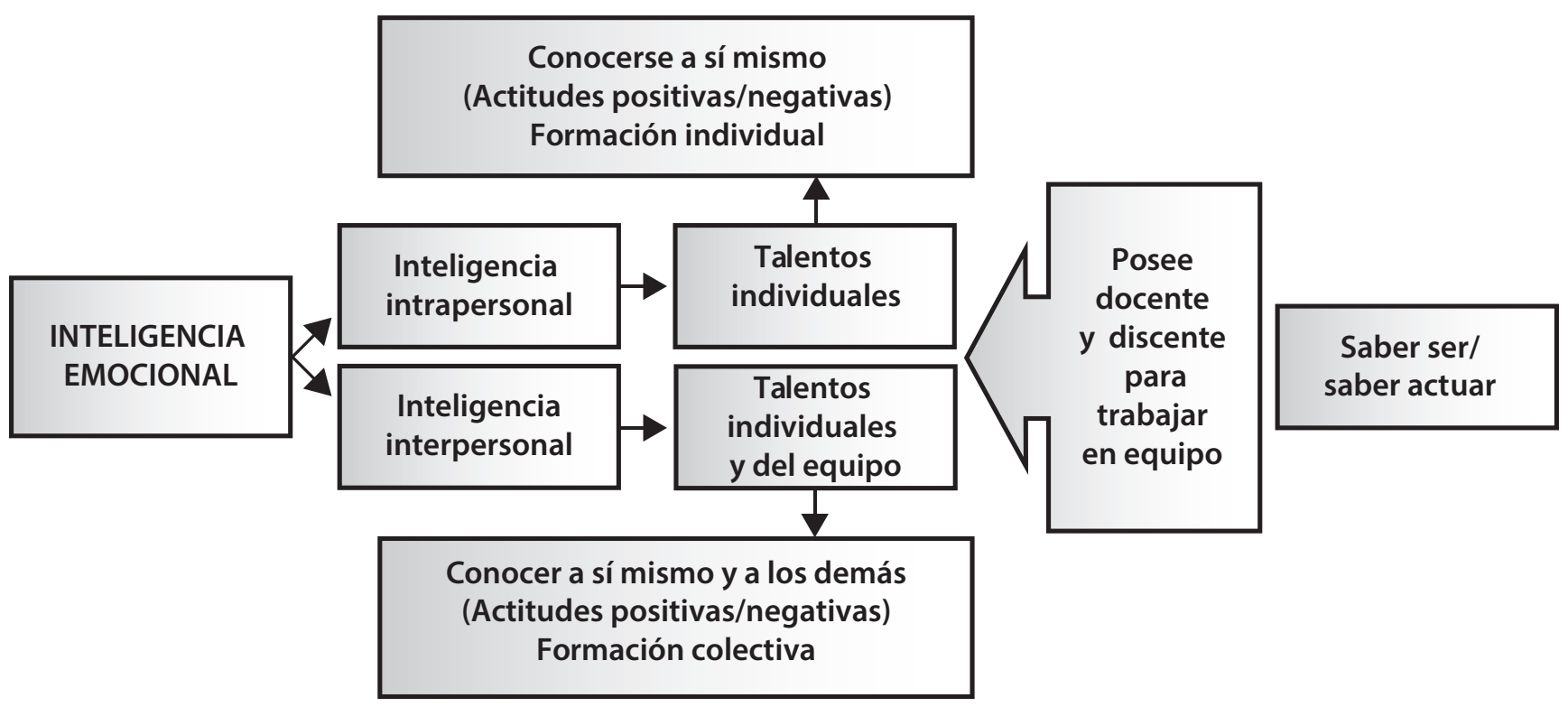

Elaboración propia: Rivadeneira (2013). Adaptado Goleman (1995), Gardner (1995), Boterf (2005)

García (1988) expone que la educación colectiva y la educación individual son formas parciales e incompletas de educación. Es decir, la educación individual separa el sujeto de los compañeros y le impide establecer relaciones sociales de igualdad, de enriquecerse a través de los trabajos en equipo, lo hace dependiente, con lo cual reduce sus posibilidades de desarrollo integral. La educación colectiva se entiende como la acción del docente que estimula, orienta y dirige la formación de un conjunto de discente. 


\section{CONCLUSIONES}

La metodologia integradora pretende el desarrollo integral del ser humano, como una persona con actitudes positivas y negativas, capacidad de autoconocimiento (inteligencia interpersonal- Goleman, 1995), en el manejo de conocer las propias emociones, y las ajenas (Inteligencia intrapersonal y interpersonal), en el motivarse y motivar a los demás, en el poder reconocer emociones ajenas y en el poder relacionarse con los demás. El propósito de la metodología integradora es orientar, guiar, fortalecer la formación de los discentes para saber ser/actuar. Para poder llevar a cabo este objetivo el docente debe hacer uso de las siguientes acciones metodológicas en el quehacer educativo:

- Fomentar el diálogo convergente, la participación activa y democrática

- Fomentar el trabajo en equipo:

- Conocer los valores positivos y negativos del equipo y su grado de participación

- Fomentar la visión integradora del equipo

- Fomentar la participación, colaboración, implicación de todo los integrantes

- Incentivar el desarrollo de cada miembro del equipo

- Facilitar la co-autoevaluacion de las habilidades individuales y grupales

- Ofrecer retroalimentación.

- Fomentar desde las diferentes disciplinas del conocimiento una cultura de valores positivos, que unifique los saberes conceptuales, procedimentales y actitudinales
- Reconocer los valores negativos para desarrollar habilidades para suavizar expresiones: ira, furia, irritabilidad, es importante en las relaciones interpersonales

- Involucrar al discente en la construcción del conocimiento

- Respetar los estilos de pensamientos, de aprendizaje, comportamiento, personalidad

- Destacar y aceptar que cada discente es un ser humano "potencialmente talentoso", y que es capaz de aprender a aprender con la orientación de un docente mediador el método adecuado

- Desarrollar en cada discente el autoconocimiento de sus potencialidades, autovaloración, autorespeto, autodominio, autoconcepto (escala de valoración) para conseguir el desarrollo de la autoestima emocional (interna/externa).

De hecho, solamente una educación centrada en el ser humano, mediante el desarrollo de sus capacidades cognitivas, procedimentales $y$ actitudinales enmarcadas en el autoconocimiento de sus potencialidades, conocimiento de sí mismo y de los demás, en un clima favorable de comprension, diálogo convergente y apoyándose en el desarrollo de habilidades para interactuar con los candados mentales (Calderón, Hess, Pérez, 1998)

\section{BIBLIOGRAFÍA}

Aldaz, N. (2007): La inteligencia emocional aplicada en el aula de clase. Ecuador

Bar, G. (1999). Perfil y competencias del docente en el contexto institucional. Disponible en internet: http:// educacion.jalisco.gov.mx/consulta/educar/05.html

Barreiro, A. (2003): Análisis de las competencias profesionales de los orientadores escolares. España. Tesis Doctoral

Bateman y Crant (2005): Proactividad y éxito en el trabajo. 
Braslaysky, C. (1998): Bases. Orientación y criterios para el diseño de programas de formación de profesores. Biblioteca de la OEI.

Calvis, R. (2007): De un perfil tradicional a un perfil docente basado en competencias. Acción pedagógica. N. 16.

Coleman, D. (2000). LeadershipThat Gets Results. Harvard Business Review

Coll, C, y Miras, M. (1990): Características individuales y condiciones de aprendizaje: la búsqueda de interacciones, en C. Coll et al. (eds.) Desarrollo psicológico y educación. Tomo II: Psicología de la Educación. Madrid: Alianza

Coll, C. Miras, M. (1990): La representación mutua/alumno y sus repercusiones sobre la enseñanza - aprendizaje. En C. Coll, J. Palacios y A. Márchense (Comp.). Desarrollo psicológico y educación II. Madrid.

Delors, J. (1996): Informe a la UNESCO de la Comisión sobre educación para el siglo XXI. La educación un tesoro. Madrid. Disponible en internet: www.monografias.com

Ekman, P. (1999): Basic Emotions. In T. Dalgleish and T. Power New York

Fierro, J. (2003): La práctica docente y sus dimensiones. Valorar. UG. Disponible en internet: www.google.es

Fredrickson, L. (1998): What good are positive emotions? Review. Of General Psychology

Fredrickson, L. (2001): The role of positive emotions. In positive psychology: The Broaden and build theory of positive emotions. American Psychologist.

Gadner, H. (2003): Las inteligencias múltiples. Paidos. Barcelona

García, J. (2005): Glosario de términos Básicos en Regulación y Acreditación. Educación Superior. España
García, V. (1988): Educación Personalizada. Grupo editor quinto centenario S.A. Bogotá

Glasser, W. (1984): Foreword. En B. Green (comp). New paradigms for creating quality schools. Chapel Hill: New View Publications.

Guimeno, J. (2004): El sistema educativo: una mirada crítica.

Ibarra, A. (2005): Formación de Recursos Humanos y Competencia Laboral. Cinterfor. Boletín OTI n. 149. Montevideo

Irigain, M. y Vargas, F. (2002): Competencia laboral: manual de conceptos, métodos y aplicación en el sector salud. Montevideo.

Le Boterf, G. (2001): Ingineria de las competencias. Barcelona

Marín, R. (1976): Valores, objetivos y actitudes en la educación. Miñón. España

McLeod, B. (1989): Beliefs, attitudes, and emotions: new vie of affect in mathematics education. En McLeod y Adams (Eds). New York

McCombs, L. y Whisler S. (1997): The learner-centered clashroom and school. San Francisco: Jossey-Bass. (Trad. Cast. La clase y la escuela centradas en el aprendiz. Paidos. Barcelona.

Miras, M. (2001): Afectos, emociones, atribuciones y expectativas: el sentido del aprendizaje escolar. En C. Coll, J. Palacios y A. Marchesi (Comps.). Desarrollo psicológico y educación II. Psicología de la Educación (pp.309-330). Madrid: Alianza

Morin, E. (2000) A cabeça bem feita: Repensar a reforma, reformar o pensamiento. Ed. Bertrand Brasil, Río de Janeiro.

Tedesco, J. (1995): El nuevo pacto educativo. Anaya. España 